\title{
PARÂMETROS FISIOLÓGICOS EM FUNÇÃO DA ESPÉCIE DE CAFÉ E DA LUMINOSIDADE NATURAL
}

\author{
José Fernandes Neto ${ }^{1}$ \\ José Laércio Favarin ${ }^{2}$ \\ Marcos Silveira Bernardes ${ }^{2}$ \\ Durval Dourado-Neto ${ }^{2,3}$
}

\section{RESUMO}

Foi realizada uma pesquisa exploratória com o objetivo de avaliar a influência do nível de luminosidade sobre parâmetros fisiológicos em mudas de café em condições de viveiro. Foram estudadas as espécies de café Coffea arabica e Coffea dewevrei, conduzidas até o sexto par de folhas com $50 \%$ de luz (padrão para mudas). Posteriormente, as plantas foram divididas em dois lotes e mantidas sob $30 \% \pm 5 \%$ e a $100 \%$ de luz durante 60 dias para adaptação às condições de luminosidade. As determinações da taxa de fotossintese, condutância estomática e taxa de transpiração foliar foram efetuadas no primeiro par de folhas completamente expandidas no ápice do caule, realizadas às $7 \mathrm{~h} 30,9 \mathrm{~h} 30,11 \mathrm{~h} 30$, $13 \mathrm{~h} 30,15 \mathrm{~h} 30$ e $17 \mathrm{~h} 30$ horas. Durante as avaliações determinaram-se os parâmetros do ambiente, como radiação solar, temperatura do ar e déficit de pressão de vapor, bem como da planta, a temperatura foliar. As espécies estudadas apresentaram comportamento semelhante para a fotossíntese em relação aos níveis de luminosidade expostas. A condutância estomática (gs), em alguns períodos, foram maiores nas plantas da cultivar da espécie Coffea arabica, independentemente dos níveis de luz, havendo correlação entre essa variável (gs) com a taxa fotossintética. A transpiração variou com a espécie, principalmente, em função da condutância estomática.

1 Aluno de Pós-graduação, Departamento de Produção Vegetal, USP/Esalq.

2 Prof. Departamento de Produção Vegetal, E-mail: jlfavari@esalq.usp.br

3 Bolsista do CNPq. 
Palavras-Chave: Coffea arabica, Coffea dewevrei, mudas, fotossintese, condutância estomática, transpiração

\section{ABSTRACT}

This study was carried out with the objective of evaluating the effect of light intensity on physiological paramete $\mathrm{s}$ in coffee plants under nursery conditions. The coffee especies Coffea arabica and Coffea dewevrei were studied, conducted until the sixth leaf pair with $50 \%$ of the full sunlight. Later, plants were divided in two groups kept under the two light levels of $30 \pm 5 \%$ e $100 \%$ of full sunlight during 60 days for the adaptation to the new light regime. The measurements of photosynthesis rate, stomata conductance and leaf transpiration rate were made on the first leaf pair with fully expanded leaves, at the apex of the plants, at 7:30, 9:30, 11:30, 13:30, 15:30 and 17:30. The weather parameters as solar radiation, air temperature, and water vapor pressure and the plant parameter leaf temperature were monitored. Both species presented similar behavior in terms of photosynthesis rate in respect to light intensity. The stomata conductance (gs), in some hours, was greater for the species Coffea arabica, regardless the light regime, occurring a correlation between this variable (gs) and photosynthesis rate. The transpiration varied with the coffee species, according to the stomata conductance.

Key words: Coffea arabica, Coffea dewevrei, planting material, photosynthesis, stomata condutance, transpiration.

\section{INTRODUÇÃO}

As espécies de café distribuem-se ao longo da região tropical central do Continente Africano, apresentando grande variabilidade em relação às características morfológicas de folhas, flores e frutos, bem como em relação aos caracteres agronômicos, fisiológicos e bioquímicos.

Os mecanismos fisiológicos que diferenciam as plantas entre as espécies adaptadas à sombra e ao sol ainda não estão devidamente esclarecidos. As folhas de espécies de sombra, como os cafeeiros arábica, 
geralmente possuem maior proporção de clorofila na matéria seca e menor quantidade dessa substância por unidade de área foliar. $\mathrm{O}$ alto conteúdo de clorofila em folhas de plantas adaptadas ao sombreamento, combinada com a redução da espessura da folha são características que tornam as plantas mais eficientes na captação da energia luminosa. Em algumas plantas, como o cafeeiro, a eficiência quântica de folhas sombreadas, geralmente, é maior que nas folhas expostas ao sol (Kozlowski et al., 1991).

Nutman (1937) verificou que a taxa de assimilação das folhas de café diminui significativamente quando expostas à luz solar, especialmente no período das 9 h00 às 14 h00 horas. Em dias com baixa nebulosidade a taxa de assimilação de folhas individuais foi três vezes superior sob sombreamento moderado do que em plantas expostas ao sol. A explicação para o efeito da radiação solar na taxa de assimilação foi relacionada com a sua ação na abertura dos estômatos. Em condições de sombreamento os estômatos permanecem abertos o dia todo, enquanto nas plantas expostas ao sol permanecem fechados parte do dia, predominantemente, das 10 h00 às 16 h00 horas.

Estudando os níveis de luminosidade de $25 \%, 50 \%$ e $100 \%$ da luz natural em mudas de café, Maestri \& Gomes (1961) concluíram que a área foliar total, a área média das folhas e o número médio de folhas por planta aumentaram com a diminuição da quantidade de luz, ocorrendo os valores menores em cafeeiros a pleno sol.

No estudo de crescimento de mudas de cafeeiro sob quatro níveis de luz, Silveira \& Maestri (1973) concluíram que o maior crescimento, avaliado pela matéria seca total da planta, foi verificado para o nível de $50 \%$ de luminosidade. Fahl et al. (1994) estudaram o efeito da luminosidade de $30 \%, 50 \%$ e $100 \%$ da luz nas respostas morfológicas e fisiológicas como área foliar, altura da planta, teor de clorofila, de nitrogênio total e atividade da redutase de nitrato em vários cultivares de café,. Os resultados evidenciaram que essas variáveis foram influenciadas, diferentemente, pelos níveis de luz. O cultivar Apoatã IAC-3597 (Coffea canephora) apresentou o melhor desenvolvimento quando cultivado a $50 \%$ de luz, ao passo que os cultivares de C. arabica (Catuaí IAC 81 e 
Mundo Novo IAC 388-17) não tiveram o seu crescimento alterado nos níveis de $50 \%$ e $100 \%$ de luz. O maior sombreamento, a $30 \%$ de luz, comparado ao cultivo a pleno sol, reduziu o crescimento das plantas dos cultivares de $C$. arabica, mas não o modificou para a espécie $C$. canephora.

De acordo com Freitas (2000), que estudou o efeito de $30 \%, 50 \%$, $70 \%$ e $100 \%$ da luz natural em cultivares de Coffea arabica, as plantas mantidas a $30 \%$ de luminosidade apresentaram as maiores taxas de fotossíntese, não constatando diferenças entre cultivares dessa espécie.

A espécie $C$. dewevrei apresenta características de grande interesse para o melhoramento vegetal, como a resistência à ferrugem (Hemileia vastatrix), tolerância a nematóides e bicho mineiro (Leucoptera coffeella), problemas fitossanitários que afetam, significativamente, o rendimento do cafeeiro (Aguiar, 2001). Apesar da importância dessa espécie para os programas de melhoramento genético, não há pesquisas exploratórias sobre o seu comportamento e sua fisiologia.

Este trabalho foi realizado com o objetivo de avaliar, comparativamente, a influência da luminosidade nos parâmetros fisiológicos como a taxa fotossintética, condutância estomática e transpiração foliar em mudas de cafeeiros das espécies $C$. arabica (população controle) e $C$. dewevrei em condições de viveiro.

\section{MATERIAL E MÉTODOS}

O experimento foi conduzido no Departamento de Produção Vegetal da Esalq, Universidade de São Paulo, Piracicaba, SP.

As mudas das espécies $C$. arabica e $C$. dewevrei foram cedidas pelo Instituto Agronômico de Campinas, tendo sido formadas de acordo com a recomendação oficial, em ambiente com $50 \%$ de luz (Thomaziello et al., 2000).

O cultivar Mundo Novo IAC 388-17 da espécie C. arabica foi escolhido como população controle, devido a sua ampla distribuição nas diferentes regiões cafeeiras e pela sua importância econômica na cafeicultura. A espécie $C$. dewevrei foi utilizada pelas suas características agronômicas de interesse para programas de melhoramento genético, visando à introdução de resistência à ferrugem (Hemileia vastatrix), tolerân- 
cia a nematóides e bicho mineiro (Leucoptera coffeella) nos cultivares arábica. Para efeito de comparação, foram utilizadas mudas com a mesma idade fisiológica, apresentando o mesmo número de pares de folhas emitidas. Trinta mudas de cada espécie estudada foram transplantadas para recipientes plásticos com capacidade de 8 litros e colocadas sob cobertura com sombrite a $50 \%$ de luminosidade para a recuperação do estresse sofrido com o transplantio.

Posteriormente, as mudas foram separadas em dois lotes de quinze plantas de cada espécie, submetidos a dois níveis de luminosidade 30 $\pm 5 \%$ e $100 \%$ de luz, permanecendo nessas condições por um período de 60 dias, visando à sua adaptação. A partir desse período, foram iniciadas as avaliações das variáveis: (i) taxa de fotossíntese (A); (ii) condutância estomática (gs); (iii) transpiração foliar (E). Durante as determinações das variáveis foram, também, monitoradas as condições do ambiente, como radiação solar (RS), temperatura do ar (Tar) e déficit de pressão de vapor (DPV), bem como as condições das plantas, como a temperatura foliar (Tf).

As determinações das variáveis foram realizadas na primeira fotha completamente expandida a partir do ápice do caule, as $7 \mathrm{~h} 30,9 \mathrm{~h} 30$, $11 \mathrm{~h} 30,13 \mathrm{~h} 30,15 \mathrm{~h} 30$ e $17 \mathrm{~h} 30$ horas, utilizando um medidor portátil de fotossintese modelo LI - 6400 (LICOR NE, USA).

Adotou-se o delineamento experimental inteiramente casualizado, com quatro repetições, em esquema fatorial $(2 \times 2)$, obtidos pela combinação de dois níveis de luminosidade $30 \% \pm 5 \%$ e $100 \%$ da luz natural e duas espécies de café: $C$. arabica e C. dewevrei.

\section{RESULTADOS E DISCUSSÃO}

Na Tabela 1 são apresentados os resultados determinados a partir das $7 \mathrm{~h} 30$ horas até às $17 \mathrm{~h} 30$ horas para as variáveis taxa de fotossíntese, condutância estomática e transpiração foliar de folhas específicas na planta.

A fotossíntese verificada às $7 \mathrm{~h} 30$ horas da manhã nas plantas a pleno sol é superior $(\mathrm{p}<0,01)$ àquela determinada nas plantas a $30 \% \pm 5$ $\%$ de luz, cujas taxas foram iguais a $4,53 \mu \mathrm{mol} \mathrm{CO} \mathrm{m}^{-2} \mathrm{~s}^{-1}$ e $3,51 \mu \mathrm{mol}$ $\mathrm{CO}_{2} \mathrm{~m}^{-2} \mathrm{~s}^{-1}$ para as espécies C. arabica (cv. Mundo Novo IAC 388-17) e 
C. dewevrei, respectivamente. As taxas determinadas, nesse período, não variaram em função da espécies estudadas. A maior taxa fotossintética não é explicada pelo comportamento da condutância estomática (gs), uma vez que esta variável não diferiu para o fator nível de luminosidade, independentemente da espécie. Entre os fatores do ambiente, o único parâmetro que, provavelmente, influenciou na diferença observada para a taxa fotossintética, foi a quantidade de radiação solar (RS) que as plantas ficaram expostas nesse período, tendo sido determinados valores iguais a $1.477 \mu \mathrm{mol}$ quanta $\mathrm{m}^{-2} \mathrm{~s}^{-1}$ (C. arabica) e $1.381 \mu \mathrm{mol}$ quanta $\mathrm{m}^{-2} \mathrm{~s}^{-1}(C$. dewevrei) enquanto, nessa ordem, os valores determinados a $30 \% \pm 5 \%$ de luz foram de 392 e $395 \mu \mathrm{mol}$ quanta $\mathrm{m}^{-2} \mathrm{~s}^{-1}$ (Tabela 2). O déficit de pressão de vapor (DPV), em valores absolutos, foi maior nas condições a pleno sol $(2,37 \mathrm{KPA})$ do que em relação às plantas sombreadas $(2,10$ KPA) para o cultivar da espécie C. arabica e para a espécie C. dewevrei os valores determinados eram de 2,54 e $1,88 \mathrm{KPA}$ a pleno sol e $30 \pm 5 \%$ de luz, respectivamente.

Não foram detectadas diferenças significativas para as variáveis condutância estomática (gs) e transpiração foliar (E) em função da espécie e para a interação delas com a quantidade de luz.

Nos períodos das 9 h30 horas e 11 h30 horas não foram detectadas diferenças para as variáveis avaliadas, entre as espécies a pleno sol e a 30 $\% \pm 5 \%$ de luz, bem como para a interação entre elas e o nível de luminosidade. A ausência de significância entre as variáveis avaliadas nas plantas a pleno sol deve-se, provavelmente, ao excesso de irradiância sobre elas, cujos valores variaram de 1.951 a $2.211 \mu \mathrm{mol}$ quanta $\mathrm{m}^{-2} \mathrm{~s}^{-1}$.

A taxa fotossintética foi também superior $(p<0,05)$ para o fator luminosidade às $13 \mathrm{~h} 30$ horas, quando a fotossíntese determinada nas plantas submetidas a $30 \pm 5 \%$ de luz atingiu valores iguais a $2,32 \mu \mathrm{mol}$ quanta $\mathrm{m}^{-2} \mathrm{~s}^{-1} \mathrm{em}$ ambas as espécies, conforme constatado por Freitas (2000), em estudo com vários cultivares de arábica. A pleno sol as plantas apresentaram taxas fotossintéticas de 0,67 e 1,76 $\mu \mathrm{mol}$ quanta $\mathrm{m}^{-2} \mathrm{~s}^{-1}$ para as espécies C. arabica e C. dewevrei, respectivamente (Tabela 1). Esse comportamento do cultivar da espécie $C$. arabica deve-se a maior condutância estomática $(\mathrm{p}<0,05)$ que, no período foi igual a $0,18 \mathrm{~mol} \mathrm{~m}^{-2} \mathrm{~s}^{-1}$, quando a 
pleno sol a condutância estomática foi duas vezes menor, apresentando valor igual a $0,09 \mathrm{~mol} \mathrm{~m}^{-2} \mathrm{~s}^{-1}$ no mesmo nível de luminosidade, corroborando a afirmação de que a espécie $C$. arabica tem um controle estomático que se verifica mais rapidamente (Da Matta et al., 1997), superior, inclusive, em relação à espécie $C$. dewevrei, conforme evidenciado nesse experimento. A condutância estomática é uma medida realizada nas folhas e dá uma idéia da resistência à abertura estomática; assim, maiores valores para a condutância estomática significam menor resistência à penetração de $\mathrm{CO}_{2}$ no mesófilo foliar.

A maior abertura estomática, representada pela maior condutância, explica também a maior perda de água $(\mathrm{p}<0,05)$ via transpiração foliar $\left(6,04 \mathrm{mmol} \mathrm{m}^{-2} \mathrm{~s}^{-1}\right)$ em relação à quantidade determinada para a espécie $C$. dewevrei $\left(3,90 \mathrm{mmol} \mathrm{m}^{-2} \mathrm{~s}^{-1}\right)$ nas mesmas condições de luz $(30 \% \pm 5 \%)$. Embora não tenha sido constatada diferença para a taxa fotossintética as 13 h30 horas entre as espécies a pleno sol, verifica-se que o valor absoluto para essa variável na espécie $C$. dewevrei $\left(1,76 \mu \mathrm{mol} \mathrm{CO}_{2} \mathrm{~m}^{-2} \mathrm{~s}^{-1}\right)$ é, aproximadamente, 2,7 vezes superior àquela verificada na espécie $C$. arabica $\left(0,67 \mu \mathrm{mol} \mathrm{CO}_{2} \mathrm{~m}^{-2} \mathrm{~s}^{-1}\right)$.

Nas condições a pleno sol as $13 \mathrm{~h} 30$ horas, embora não tenha sido detectada diferença para a variável condutância estomática (gs) entre as espécies, a resistência à penetração do $\mathrm{CO}_{2}$, em valores absolutos, foi 2,3 vezes inferior para a espécie $C$. dewevrei uma vez que a condutância foi de $0,14 \mathrm{~mol} \mathrm{~m}^{-2} \mathrm{~s}^{-1}$ o que, provavelmente, explica a maior transpiração foliar $(p<0,05)$ para essa espécie $\left(5,43 \mathrm{mmol} \mathrm{m}^{-2} \mathrm{~s}^{-1}\right)$ em comparação a transpiração constatada na espécie $C$. arabica $\left(2,37 \mathrm{mmol} \mathrm{m}^{-2} \mathrm{~s}^{-1}\right)$, pois em valor absoluto a condutância estomática foi bem menor e igual a 0,06 mol $\mathrm{m}^{-2} \mathrm{~s}^{-1}$, evidenciando maior adaptabilidade daquela espécie $(C$. dewevrei) ao plantio a pleno sol. A maior taxa de transpiração para a espécie $C$. dewevrei a pleno sol não pode ser explicada pelo déficit de pressão vapor (DPV), uma vez que nessas condições (100\% de luz) o déficit de pressão de vapor foi equivalente a 3,57 KPA, enquanto sob sombramento (30 $\pm 5 \%$ luz) ele foi de 4,15 KPA. Segundo Pilar et al. (1989) a condutância estomática é, frequentemente, correlacionada com o déficit de pressão de vapor, observação que não foi confirmada pelos 
Tabela 1. Valores obtidos para as variáveis fotossíntese $\left(\mathrm{A}, \mathrm{mmol} \mathrm{CO}_{2}\right.$ $\mathrm{m}^{-2} \mathrm{~s}^{-1}$ ), condutância estomática ( $\mathrm{gs}, \mathrm{mol} \mathrm{m}^{-2} \mathrm{~s}^{-1}$ ) e transpiração

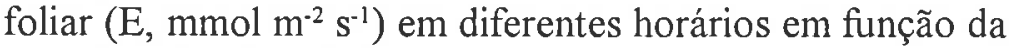
espécie e do nível de luminosidade.

\begin{tabular}{|c|c|c|c|c|c|c|c|c|}
\hline Variável & Espécie & Nível luz & $7 \mathrm{~h} 30$ & $9 h 30$ & $11 \mathrm{~h} 30$ & $13 \mathrm{~h} 30$ & $15 \mathrm{~h} 30$ & $17 \mathrm{~h} 30$ \\
\hline \multirow{5}{*}{ A } & \multirow{2}{*}{ C. arabica } & $100 \%$ & $4,53^{* *(L)}$ & 1,78 & 2,70 & 0,67 & $2,47^{* *(L)^{*}(I)}$ & 0,087 \\
\hline & & $30 \pm 5 \%$ & $-1,19$ & 1,86 & 2,70 & $2,32^{* *(L)}$ & 0,05 & $-0,103$ \\
\hline & \multirow{3}{*}{ C. dewevrei } & $100 \%$ & $3,51^{* *(L)}$ & 1,74 & 2,30 & 1,76 & $1,39^{* *(L) *(I)}$ & $-0,103$ \\
\hline & & & & & & & & \\
\hline & & $30 \pm 5 \%$ & $-1,52$ & 1,43 & 1,86 & $2,32^{* *(L)}$ & 0,28 & $-0,940$ \\
\hline \multirow{4}{*}{ gs } & \multirow{2}{*}{ C. arabica } & $100 \%$ & 0,26 & 0,12 & 0,14 & 0,06 & $0,11^{* *(L)}$ & 0,04 \\
\hline & & $30 \pm 5 \%$ & 0,29 & 0,18 & 0,22 & $0,18^{*(1)}$ & 0,01 & 0,03 \\
\hline & \multirow[b]{2}{*}{ C. dewevrei } & $100 \%$ & 0,26 & 0,15 & 0,21 & 0,14 & $0,07^{* *(L)}$ & 0,04 \\
\hline & & $30 \pm 5 \%$ & 0,34 & 0,15 & 0,15 & 0,09 & 0,03 & 0,01 \\
\hline \multirow{4}{*}{$\mathrm{E}$} & \multirow[b]{2}{*}{ C. arabica } & $100 \%$ & 5,74 & 4,71 & 4,95 & 2,37 & $4,10^{* *(L))^{*}(1)}$ & 1,24 \\
\hline & & $30 \pm 5 \%$ & 5,96 & 5,76 & 6,84 & $6,04^{*(1)}$ & 0,55 & 0,94 \\
\hline & \multirow[b]{2}{*}{ C. dewevrei } & $100 \%$ & 6,08 & 5,46 & 6,96 & $5,43^{*(1)}$ & $2,94^{* *(L) *(I)}$ & 1,38 \\
\hline & & $30 \pm 5 \%$ & 6,89 & 4,88 & 5,52 & 3,90 & 1,31 & 0,51 \\
\hline
\end{tabular}

* Significativo ao nivel de $5 \%$ de probabilidade pelo teste $\mathrm{F}$ ** Significativo ao nível de $1 \%$ de probabilidade pelo teste $\mathrm{F}$

(L) Significância para o fator luz (1) significância para a interação entre a espécie e o nivel de luz

resultados deste experimento (Tabela 2 ). No período das $13 \mathrm{~h} 30$ horas (30 $\% \pm 5 \%$ de luz), a condutância estomática foi igual a $0,18 \mathrm{~mol} \mathrm{~m}^{-2} \mathrm{~s}^{-1}$ para um déficit de pressão de 3,81 KPA no cultivar da espécie $C$. arabica (cv. Mundo Novo IAC 388-17), enquanto para a espécie C. dewevrei os valores dessas variáveis foram iguais a $0,09 \mathrm{~mol} \mathrm{~m}^{-2} \mathrm{~s}^{-1}$ (gs) e 4,15 KPA (DPV). 
O cultivar da espécie $C$. arabica estudado apresentou a pleno sol (13h30 horas) os estômatos praticamente fechados, refletindo menor $(\mathrm{p}<0,05)$ taxa de transpiração $\left(2,37 \mathrm{mmol} \mathrm{m}^{-2} \mathrm{~s}^{-1}\right)$ em relação àquela verificada na espécie $C$. dewevrei $\left(5,43 \mathrm{mmol} \mathrm{m}^{-2} \mathrm{~s}^{-1}\right)$, ocorrendo comportamento contrário entre as espécies sob sombreamento. Esse resultado reforça as evidências de que a espécie $C$. dewevrei é, comparativamente, mais adaptada a pleno sol.

No período das $15 \mathrm{~h} 30$ horas foi observada diferença altamente significativa em relação ao fator luminosidade, para a taxa fotossintética (A), condutância estomática $\left(\mathrm{g}_{\mathrm{s}}\right)$ e taxa de transpiração foliar $(\mathrm{E})$, enquanto para a interação entre as espécies e o nível de luminosidade houve diferença $(\mathrm{p}<0,05)$ para as variáveis taxa fotossintética $(\mathrm{A})$ e taxa de transpiração foliar (E). Nesse período, os valores determinados para o déficit de pressão de vapor (DPV) foram os mais elevados durante as avaliações. Para a espécie $C$. dewevrei a pleno sol o déficit de pressão de vapor alcançou valor igual a $4,20 \mathrm{KPA}$, enquanto sob sombreamento (30\% $55 \%$ luz) foi de 4,38 KPA, bem como em relação ao cultivar da espécie C. arabica (cv. Mundo Novo IAC 388-17), nas mesmas condições de exposição (30 \% $\pm 5 \%$ luz), em que o déficit de pressão de vapor alcançou 4,44 KPA.

As avaliações realizadas às $15 \mathrm{~h} 30$ horas apresentaram maior coerência dos resultados obtidos nesse experimento. A maior taxa fotossintética $(p<0,01)$ foi observada a pleno sol $(100 \%$ de luz) na espécie C. arabica, que alcançou $2,47 \mu \mathrm{mol} \mathrm{CO}_{2} \mathrm{~m}^{-2} \mathrm{~s}^{-1}$, enquanto, nas mesmas condições, a espécie $C$. dewevrei apresentou taxa equivalente a 1,39 $\mu \mathrm{mol} \mathrm{CO} \mathrm{m}^{-2} \mathrm{~s}^{-1}$. Observa-se também que houve uma inversão em relação ao período anterior, quando as maiores taxas fotossintéticas foram determinadas nas plantas submetidas a $30 \pm 5 \%$ da luz natural.

As maiores taxas fotossintéticas a pleno sol, para ambas as espécies as $15 \mathrm{~h} 30$ horas, podem ser explicadas pela maior condutância estomática $(p<0,01)$ cujos valores obtidos foram iguais a 0,11 e 0,07 mol m $\mathrm{m}^{-2} \mathrm{~s}^{-1}$ nas espécies $C$. arabica e $C$. dewevrei, respectivamente. Não se observou diferença em função da espécie para a variável condutância estomática, apresentando ambas o mesmo comportamento estomático. 
Tabela 2. Valores relativos ao ambiente como temperatura do ar (Tar, ${ }^{\circ} \mathrm{C}$ ), déficit de pressão de vapor (DPV, KPA) e radiação solar (RS, mmol quanta $\mathrm{m}^{-2} \mathrm{~s}^{-1}$ ) e à planta, como a temperatura foliar $\left(\mathrm{Tf},{ }^{\circ} \mathrm{C}\right)$ em dois níveis de luminosidade em cafeeiros das espécies Coffea arabica e Coffea dewevrei.

\begin{tabular}{lccccccccccccccc}
\hline \multirow{2}{*}{ Espécie } & Variável & \multicolumn{2}{c}{$7 \mathrm{~h} 30$} & \multicolumn{2}{c}{$9 \mathrm{~h} 30$} & \multicolumn{1}{c}{$11 \mathrm{~h} 30$} & \multicolumn{2}{c}{$13 \mathrm{~h} 30$} & \multicolumn{3}{c}{$15 \mathrm{~h} 30$} & \multicolumn{2}{c}{$17 \mathrm{~h} 30$} \\
& & $100 \%$ & $30 \%$ & $100 \%$ & $30 \%$ & $100 \%$ & $30 \%$ & $100 \%$ & $30 \%$ & $100 \%$ & $30 \%$ & $100 \%$ & $30 \%$ \\
\hline \multirow{3}{*}{ C.arabica } & Tar & 29,1 & 31,5 & 32,3 & 34,9 & 32,4 & 35,3 & 32,3 & 36,2 & 34,4 & 36,4 & 31,5 & 32,5 \\
& DPV & 2,37 & 2,10 & 3,69 & 3,08 & 3,57 & 2,92 & 4,26 & 3,81 & 3,53 & 4,44 & 2,83 & 3,14 \\
& PAR & 1477 & 392 & 1951 & 655 & 2119 & 782 & 2033 & 664 & 1510 & 544 & 164 & 41 \\
& Tfl & 30,5 & 30,0 & 35,6 & 34,3 & 35,2 & 34,8 & 36,4 & 36,5 & 35,0 & 37,1 & 31,1 & 31,9 \\
& & & & & & & & & & & & & \\
& Tar & 30,6 & 31,2 & 34,2 & 35,2 & 34,1 & 35,8 & 334,7 & 37,2 & 35,5 & 36,9 & 32,0 & 32,9 \\
& DPV & 2,54 & 1,88 & 3,73 & 3,12 & 3,10 & 3,57 & 3,57 & 4,15 & 4,20 & 4,38 & 2,91 & 3,31 \\
& PAR & 1381 & 395 & 1966 & 670 & 2211 & 828 & 2012 & 647 & 1508 & 448 & 164 & 41
\end{tabular}

Os fatores do ambiente não explicam a maior taxa de transpiração $(p<0,01)$ para essas espécies a pleno sol ( $15 \mathrm{~h} 30$ horas) tendo sido constatado, também, que essa variável foi maior $(\mathrm{p}<0,05)$ na espécie $C$. arabica $\left(4,10 \mathrm{mmol} \mathrm{m}^{-2} \mathrm{~s}^{-1}\right)$ do que na espécie $C$. dewevrei $\left(2,94 \mathrm{mmol} \mathrm{m}^{-2} \mathrm{~s}^{-1}\right)$. Tal comportamento reforça a tese de que a espécie $C$. dewevrei é mais adaptada ao sol que o cultivar da espécie C. arabica, pois nesse período a radiação solar era, aproximadamente, de $1.500 \mu \mathrm{mol}$ quanta $\mathrm{m}^{-2} \mathrm{~s}^{-1}$, semelhante as condições verificadas as $7 \mathrm{~h} 30$ horas. Apesar das pequenas variações nas condições ambientais que envolviam as espécies, pode-se afirmar que as diferenças, verificadas entre elas, devem-se às características intrínsecas às plantas.

Em geral, os resultados encontrados na literatura evidenciam a importância dos fatores do ambiente como a temperatura do ar e déficit 
de pressão de vapor, bem como da própria planta, como a temperatura foliar, sobre as variáveis estudadas, explicando, em parte, os resultados nesse período ( $15 \mathrm{~h} 30$ horas).

Ressalte-se que as avaliações foram realizadas ao longo de um periodo e são pontuais, tendo sido determinadas em folhas especificas nas plantas o que, evidentemente, não invalida a análise comparativa.

\section{CONCLUSÕES}

As espécies estudadas apresentaram comportamentos semelhantes para a fotossíntese em relação aos niveis de luminosidade expostas. A condutância estomática (gs), em alguns periodos, foi maior nas plantas do cultivar da espécie C. arabica (cv. Mundo Novo IAC 388-17), independentemente dos níveis de luz, havendo correlação entre essa variável (gs) com a taxa fotossintética. A transpiração variou com a espécie, principalmente, em função da condutância estomática.

\section{REFERÊNCIAS BIBLIOGRÁFICAS}

AGUIAR, A.T. da E., 2001. Descritores para Caracterização de Cultivares e Linhagens de Café Tipo Arábica. Campinas, 98p. Dissertação (Mestrado) - Instituto Agronômico.

FAHL, J.L.; CARELLI, M.L.C.; VEJA, J.; MAGALHÃES, A.C., 1994. Nitrogen and Irradiance Levels Affecting Net Photosynthesis and Growth of Young Coffee Plants (Coffea arabica L.). J, Hort. Sci., 69(1):161-169.

FREITAS, R.B. de, 2000.. Avaliações Ecoficiológicas de Cafeeiros (Coffea arabica L.) e Seringueiras (Hevea órasiliensis Muell Arg.) em Diferentes Sistemas de Cultivo. Lavras, 57p. Dissertação (Mestrado) - Universidade Federal de Lavras.

KOZLOWSKI, T.T.; KRAMER, P.J.; PALLARDY, S.G., 1991. The Physiological Ecology of Woody Plants. Academic Press Inc., 657p.. KUMAR, D.; TIESZEN, L.L., 1980. Photosynthesis in Coffea arabica L. Effects of Light and Temperature. Experimental Agriculture, 16(1):13-19. 
MAESTRI, M.; GOMES, F.R., 1961. Crescimento de Mudas de Café (Coffea arabica L. cv Bourbon) sob Diferentes Niveis de Luz. Revista Ceres, 10(59):324-331.

NUTMAN, F.J., 1937. Studies of the Physiology of Coffea arabica. Photosynthesis of Coffee Leaves Under Natural Conditions. Annals of Botany, 1:353-367.

SILVEIRA, A.J.; MAESTRI, M., 1973. Cresciniento de Mudas de Café (Coffea arabica L. cv Bourbon) sob Quatro Níveis de Luz. Revista Ceres, 20(111):354-369.

THOMAZIELLO, R.A.; FAZUOLI, L.C.; PEZZOPANE, J.R.M.; FAHL, J.I.; CARELLI, M.L.C., 2000. Café Arábica: Cultura e Técnicas de Produção. Campinas: Instituto Agronômico, 82p. (Boletim Técnico, 187). 